\title{
O efeito do crioalongamento e da massoterapia no trismo pós-neoplasia bucal
}

\author{
The impacts of cryotherapy and massage in post buccal-neoplasm trismus
}

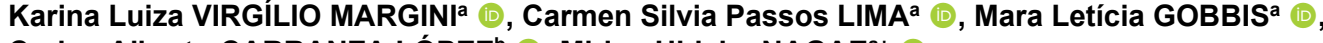 \\ Carlos Alberto CARRANZA LÓPEZ ${ }^{\mathrm{b}}$ (1), Mirian Hideko NAGAE* ${ }^{\mathrm{a} *}$ \\ aUNICAMP - Universidade Estadual de Campinas, Faculdade de Ciências Médicas, Campinas, SP, Brasil \\ bUFJF - Universidade Federal de Juiz de Fora, Departamento de Ciências Básicas da Vida, Governador Valadares, MG, \\ Brasil
}

\begin{abstract}
Como citar: Virgílio Margini KL, Lima CSP, Gobbis ML, Carranza López CA, Nagae MH. 0 efeito do crioalongamento e da massoterapia no trismo pós-neoplasia bucal. Rev Odontol UNESP. 2020;49:e20200026. https://doi.org/10.1590/18072577.02620
\end{abstract}

\begin{abstract}
Resumo
Introdução: 0 trismo é uma restrição na abertura bucal de até 3,5 cm e é um dos efeitos colaterais mais comuns da radioterapia na região da cabeça e pescoço. Tal condição afeta funções cotidianas simples, como: mastigar, deglutir, falar e até exercer higiene bucal, acarretando danos não só físicos como emocionais aos indivíduos acometidos. Evidencia-se assim a necessidade da busca de tratamentos que revertam ou atenuem tal quadro. Objetivo: 0 objetivo do estudo é investigar o comportamento dos músculos masseter e supra-hioideos durante a deglutição antes e após o crioalongamento associado à massoterapia, em pacientes pós-neoplasia bucal com trismo devido à radioterapia. Material e método: A amostra final foi constituída por oito sujeitos, com faixa etária entre 40 e 64 anos, gêneros masculino e feminino, com abertura bucal menor ou igual a 3,5 cm. Para a realização da pesquisa, foram realizadas duas avaliações eletromiográficas, uma no início e outra no final do tratamento. Com 15 atendimentos, uma vez por semana, com manobras de crioalongamento associado à massoterapia nos músculos masseter e grupo muscular dos supra-hioideos. Resultado: Após a intervenção com crioalongamento e massoterapia, foi observada diferença significativa apenas no comportamento do grupo muscular dos supra-hioideos, fato não constatado no músculo masseter. Conclusão: Foi encontrada diferença significativa no comportamento do grupo muscular supra-hioideo e melhora na abertura de boca dos sujeitos. Contudo, os resultados deste estudo devem ser confirmados em casuísticas maiores.
\end{abstract}

Descritores: Trismo; neoplasias bucais; crioterapia; massagem.

\begin{abstract}
Introduction: Trismus is a restriction in the buccal opening up to $3.5 \mathrm{~cm}$ and is one of the most frequent side effect of head and neck regions radiotherapy. This condition affects simple daily activities such as chewing, swallowing speaking and even buccal hygiene, leading to not only physical but emotional damages to the subjects. This puts in evidence the need to find treatments to revert or soften this condition. Objective: This study investigates the behavior of masseter and suprahyoid muscles during swallowing before and after the cryotherapy associated to the massage in patients post buccal neoplasm with trismus due to radiotherapy. Material and method: The final sample was composed of 8 subjects, aged between 40 an 64 years, both female and male with buccal opening equal to or smaller than $3.5 \mathrm{~cm}$. Two electromyographic evaluations were conducted to perform this research, one in the beginning and one in the end of the treatment. The study was composed of 15 treatment sessions, once a week, consisting of cryotherapy maneuvers and massage in masseters and supra-hyoids muscle groups. Result: There was a significant difference in the behavior of the supra-hyoid muscles group, this fact was not observed in the masseter muscles group. Conclusion: The study concluded that after the intervention with cryotherapy and massage sessions, was observed a significant difference in the behavior of the upperhyoid muscles group and an improvement of the subjects' mouth opening capacity. However, the results of this study must be confirmed in a larger sample size.
\end{abstract}

Descriptors: Trismus; mouth neoplasms; cryotherapy; massage. 


\section{INTRODUÇÃO}

0 interesse por tratamentos relacionados às sequelas decorrentes de doenças devastadoras como a neoplasia orofaríngea tem aumentado em razão da morbidade a elas atribuída. Prejuízos como o trismo, restrição de abertura bucal em função da radioterapia, são observados entre 5 e $50 \%$ dos $\operatorname{casos}^{1,2}$, e podem acentuar a fragilidade física e emocional do paciente devido à dificuldade em exercer funções cotidianas básicas, como mastigar, falar e realizar higiene bucal ${ }^{3,4}$. Seu tratamento, entretanto, nem sempre é fácil em função da complexidade do quadro².

A radioterapia, ao conter o crescimento anômalo celular, determina necrose e processos inflamatórios dolorosos locais ${ }^{5-7}$. Ações reflexas protetoras, como o travamento bucal, são então desencadeadas para que o organismo não sofra mais prejuízos, como a modificação da excitação nervosa dos receptores sensoriais localizados no periodonto, na mucosa jugal e na musculatura orofaríngea ${ }^{8}$. Esse quadro desencadeia um novo padrão de informações em nível de tronco encefálico, o qual pode ocasionar atrofia e fibrose na mucosa jugal, nas glândulas salivares e na musculatura acessória da cabeça e pescoço $0^{5,7}$ devido à restrição de movimentos.

A dificuldade de abertura bucal pode ocorrer a qualquer momento durante os 24 meses subsequentes à radioterapia ${ }^{5}$ e é insidiosa e lentamente progressiva. Os pacientes nem sempre procuram ajuda por medo ou por considerarem que a melhora ocorrerá naturalmente 9 . Mas tal fato nem sempre ocorre e, quando o trismo não é tratado a tempo, pode ser irreversível em função da consolidação da fibrose nos tecidos subcutâneos ${ }^{10,11}$.

São escassos os relatos sobre manobras terapêuticas para melhorar tal condição ${ }^{12,13}$. A sensação desagradável ou a dor, e a limitação das estruturas remanescentes são dois fatores que devem ser considerados na busca de estratégias adequadas para o tratamento ${ }^{14}$. Intervenções apenas momentâneas - com aplicações locais medicamentosas, toque por meio de espátula de madeira ${ }^{12}$ ou massagens sem critérios ou não considerando a especificidade das regiões e a intensidade dos estímulos - nem sempre são suficientes para atenuar os quadros. Muitas vezes, inclusive, se faz necessária a combinação de modalidades terapêuticas.

O crioalongamento, tratamento com gelo seguido de alongamento, é um recurso muito utilizado para bloqueio da dor, favorecimento da flexibilidade e diminuição de contratura. Inicialmente, o posicionamento intermitente do gelo sobre o tecido alterna a vasoconstrição e a vasodilatação, uma vez que, após a retirada do gelo, há um efeito oscilatório decrescentecrescente de $2{ }^{\circ} \mathrm{C}$ a $6{ }^{\circ} \mathrm{C}^{15,16}$, que faz com que a circulação sanguínea favoreça a drenagem de nutrientes para reparo e melhora do edema tecidual ${ }^{17,18}$. Por sua vez, o tecido profundo resfriado pela repetição contínua estimula mecanorreceptores que retardam a frequência de transmissão do impulso, os quais são responsáveis pela diminuição da sensibilidade dolorosa ${ }^{13,19}$, com consequente maior conforto para aplicação de outras intervenções, como a massagem.

A massoterapia, manipulação dos tecidos moles, promove a liberação e o realinhamento das fibras musculares, reduzindo a tensão muscular das áreas acometidas para, em seguida, favorecer o retorno da movimentação e evitar atrofia no tecido ${ }^{15}$. Na face, podem ser utilizados deslizamentos, movimentos lentos rotatórios firmes e manipulação digital ${ }^{11,19}$. Com a musculatura relaxada, o alongamento muscular se torna mais efetivo. No caso do trismo, movimentos de alavanca com os dedos polegar e indicador podem mensurar com maior precisão a intensidade de abertura ${ }^{8,20}$.

A crioterapia, a massoterapia e o alongamento são modalidades de baixo custo e evitam a formação de aderência na área, além de suas aplicações poderem ser controladas pelo terapeuta com feedback do paciente.

O objetivo do presente estudo é investigar a eficácia da crioterapia associada à massoterapia e ao alongamento em pacientes após neoplasia bucal com trismo devido à radioterapia. 


\section{MATERIAL E MÉTODO}

O estudo foi realizado no Ambulatório de Motricidade Orofacial do Departamento de Desenvolvimento Humano e Reabilitação da Faculdade de Ciências Médicas da Universidade Estadual de Campinas. O estudo foi aprovado pelo Comitê de Ética em Pesquisa/UNICAMP (processo ํㅜ 16916913.4.0000.5404.)

A amostra foi constituída por oito sujeitos, com faixa etária entre 40 e 64 anos, sendo seis homens e duas mulheres.

Critérios de inclusão: pacientes com queixa de trismo, abertura bucal menor ou igual a $3,5 \mathrm{~cm}^{21}$, após tratamento combinado de quimioterapia (três aplicações intravenosas de cisplatina) e radioterapia (15 sessões) para tratamento de neoplasia de cabeça e pescoço, edêntulo devido à radioterapia, em seguimento no Ambulatório de Oncologia Clínica ou no Serviço de Odontologia do Hospital das Clínicas/FCM/UNICAMP.

Critérios de exclusão: hipersensibilidade ao frio; mucosite; submetidos a tratamentos anteriores para liberação do trismo; fazendo uso de miorrelaxante, analgésico e/ou antiinflamatório; com recidiva do tumor; comorbidade não controlada; lesão na pele na região de cabeça, pescoço, face ou intraoral; dor aguda; com trismo superior a dois anos; ulcerações; necrose; artrose.

Antes de toda sessão, toda a região facial, de pescoço e intraoral foi examinada para descartar qualquer possibilidade de sinais de recidiva ou lesão na área que impossibilitariam a aplicação das técnicas.

A abertura bucal foi mensurada com paquímetro digital Digimess ${ }^{\circledR}$ posicionado entre os rebordos alveolares na região dos dentes incisivos, superior e inferior.

0 critério adotado para considerar o trismo foi o de Dijkstra et al. ${ }^{21}$, em que não há diferença entre dentados e desdentados. Os procedimentos de crioalongamento associado à massoterapia foram realizados na região do masseter e dos supra-hioideos.

No crioalongamento, combinaram-se três procedimentos: aplicação do frio, massagem e alongamento estático. Cubos de gelo com $3 \mathrm{~cm}$ de largura por $3 \mathrm{~cm}$ de comprimento envoltos em gaze foram posicionados na região do ventre dos músculos masseter e do grupo dos suprahioideos.

Massagens intermitentes com gelo, repetidas com intervalo de um a cinco segundos, de forma gradativa, sem causar desconforto ao paciente.

Após a retirada do gelo, a área era seca, levemente massageada ${ }^{1}$ com gaze e alongada por meio de um sistema de alavanca em que os dedos polegar e indicador posicionados nos rebordos alveolares na região dos dentes incisivos liberavam ligeiramente a abertura bucal de forma intermitente por 20 segundos ${ }^{17}$. As manobras com gelo foram repetidas até o paciente relatar sensação de dormência ou analgesia, por cerca de cinco minutos 8,18,22.

Foram realizadas três baterias de exercícios em cada músculo ${ }^{8,17,22}$. Após a analgesia, foram realizados exercícios de alongamento estático alternado com três repetições de contrações isométricas (contração e relaxamento) ${ }^{1,16-18 .}$.

Massoterapia: após o crioalongamento, a pele do paciente foi totalmente seca com gaze e, para iniciar a massoterapia, foi passado óleo facial na pele do paciente a fim de favorecer a massagem. Movimentos manuais circulares foram realizados no ventre dos músculos masseter e suprahioideos até o terapeuta sentir na superfície dos dedos a liberação muscular ${ }^{1}$. Em média, foram realizadas três baterias de exercícios em cada músculo com duração de cinco segundos e intervalos de dois minutos. Após a massagem, novamente a liberação da abertura bucal era favorecida pelos movimentos de alavanca pelos dedos indicador e polegar. 


\section{RESULTADOS}

As Tabelas 1 e 2 apresentam as características dos pacientes inseridos no estudo.

Tabela 1. Frequência (porcentagem) das classes e teste de Qui-quadrado para igualdade de proporções das variáveis gênero e hábitos orais

\begin{tabular}{|c|c|c|c|c|}
\hline Característica & Classe & Frequência & Porcentagem & Teste de Qui-quadrado \\
\hline \multirow{2}{*}{ Gênero } & Feminino & 2 & 25,00 & $\chi^{2}: 2,00-$ GL: 1 \\
\hline & Masculino & 6 & 75,00 & Valor-p:0,1573 \\
\hline \multirow{3}{*}{ Tabagismo } & Antes de começar o tratamento & 2 & 25,00 & $\chi^{2}: 1,00-\mathrm{GL}: 2$ \\
\hline & $\begin{array}{l}\text { Antes de começar o tratamento e } \\
\text { que continuam tabagistas }\end{array}$ & 4 & 50,00 & Valor-p:0,6065 \\
\hline & Não & 2 & 25,00 & \\
\hline \multirow{3}{*}{ Etilismo } & Antes de começar o tratamento & 4 & 50,00 & $\chi^{2}: 1,78-G L: 2$ \\
\hline & $\begin{array}{l}\text { Antes de começar o tratamento e } \\
\text { que continuam etilistas }\end{array}$ & 1 & 12,50 & Valor-p:0,4169 \\
\hline & Não & 3 & 37,50 & \\
\hline \multirow{2}{*}{ Drogadício } & Antes do tratamento & 1 & 12,50 & $\chi^{2}: 4,50-G L: 1$ \\
\hline & Não & 7 & 87,50 & Valor-p:0,0339 \\
\hline
\end{tabular}

GL: Grau de Liberdade.

Tabela 2. Médias, desvios padrão, mínimos e máximos das medidas numéricas avaliadas no estudo

\begin{tabular}{ccccc}
\hline Variáveis & Média & Desvio padrão & Mínimo & Máximo \\
\hline Idade (anos) & 57,50 & 10,30 & 39,00 & 74,00 \\
Radioterapia & 26,50 & 11,87 & 0,00 & 37,00 \\
Abertura (mm) & 341,54 & 65,67 & 180,00 & 510,00 \\
\hline
\end{tabular}

Com relação à caracterização e ao tratamento da doença, segue o Quadro 1.

Quadro 1. Caracterização da amostra em relação à doença em cada paciente

\begin{tabular}{|c|c|c|c|}
\hline Idade (anos) & Histológico & Localização & Estadiamento \\
\hline 40 & $\begin{array}{c}\text { Carcinoma epidermoide } \\
\text { moderadamente diferenciado }\end{array}$ & $\begin{array}{l}\text { Amígdala esquerda, trígono retromolar, } \\
\text { língua e assoalho oral à esquerda }\end{array}$ & $\mathrm{T}_{3} \mathrm{~N}_{2 \mathrm{~b}} \mathrm{M}_{0}$ \\
\hline 54 & $\begin{array}{l}\text { Linfoma não Hodgkin de } \\
\text { grandes células B }\end{array}$ & Palato duro e cavidade nasal & $\mathrm{T}_{2} \mathrm{~N}_{0} \mathrm{M}_{0}$ \\
\hline 51 & $\begin{array}{l}\text { Carcinoma adenoide cístico } \\
\text { moderadamente diferenciado }\end{array}$ & Mucosa jugal direita & $\mathrm{T}_{2} \mathrm{~N}_{0} \mathrm{M}_{0}$ \\
\hline 57 & $\begin{array}{c}\text { Carcinoma epidermoide } \\
\text { moderadamente diferenciado }\end{array}$ & Lábio & $\mathrm{T}_{2} \mathrm{~N}_{2 \mathrm{~b}} \mathrm{M}_{\mathrm{x}}$ \\
\hline 74 & $\begin{array}{c}\text { Carcinoma epidermoide } \\
\text { moderadamente diferenciado }\end{array}$ & Trígono retromolar à direita & $\mathrm{T}_{2} \mathrm{~N}_{\mathrm{x}} \mathrm{M}_{\mathrm{x}}$ \\
\hline 59 & $\begin{array}{c}\text { Carcinoma epidermoide bem } \\
\text { diferenciado }\end{array}$ & Assoalho bucal & $\mathrm{T}_{1} \mathrm{~N}_{0} \mathrm{M}_{0}$ \\
\hline 58 & $\begin{array}{c}\text { Carcinoma epidermoide } \\
\text { moderadamente diferenciado }\end{array}$ & Palato duro & $\mathrm{T}_{3} \mathrm{~N}_{\mathrm{x}} \mathrm{M}_{\mathrm{x}}$ \\
\hline 64 & $\begin{array}{c}\text { Carcinoma epidermoide } \\
\text { moderadamente diferenciado }\end{array}$ & Borda lateral esquerda da língua & $\mathrm{T}_{3} \mathrm{~N}_{1} \mathrm{M}_{0}$ \\
\hline
\end{tabular}

T: tumor primário - $\mathrm{T}_{1}$ : tumor com $2 \mathrm{~cm}$ ou menos em sua maior dimensão; $\mathrm{T}_{2}$ : tumor com mais de $2 \mathrm{~cm}$ e até $4 \mathrm{~cm}$ em sua maior dimensão; $\mathrm{T}_{3}$ : tumor com mais de $4 \mathrm{~cm}$ em sua maior dimensão; $\mathrm{N}$ : linfonodos regionais - $\mathrm{N}_{\mathrm{x}}$ : os linfonodos regionais não podem ser avaliados, $\mathrm{N}_{0}$ : ausência de metástase em linfonodos regionais, $\mathrm{N}_{2 b}$ : metástase em linfonodos homolaterais múltiplos, nenhum deles com mais de $6 \mathrm{~cm}$ em sua maior dimensão; M: Metástase à distância - $\mathrm{M}_{\mathrm{x}}$ : a presença de metástase à distância não pode ser avaliada; $\mathrm{M}_{0}$ : ausência de metástase à distância. 
$\mathrm{Na}$ análise da abertura da boca, foi adotado um modelo linear generalizado misto que possibilitou testar o efeito imediato da aplicação da terapia em cada sessão e o efeito de longo prazo representado na evolução da abertura bucal ao longo das sessões, assim como o efeito da interação entre os dois efeitos.

A análise de variância calculada para comparação das médias dos momentos (antes e após a administração da técnica) e considerando o efeito da sessão como covariável, nos dá fortes indícios da existência de efeito significativo da sessão de tratamento na abertura da boca $(p=0,001)$, assim como do momento ( $\mathrm{p}=0,001)$ em que a avaliação da abertura é feita de cada sessão.

Também observamos que as médias de abertura de boca foram significativamente maiores depois do tratamento $(348,17 \mathrm{~mm})$ em comparação com as médias de antes do tratamento (335,92 mm). Ademais, como não houve efeito significativo da interação, conclui-se que tal resultado persistiu em todas as sessões, o que pode ser usado para corroborar a existência de um efeito imediato da técnica e que este se mantém constante em cada uma das sessões (Figura 1).

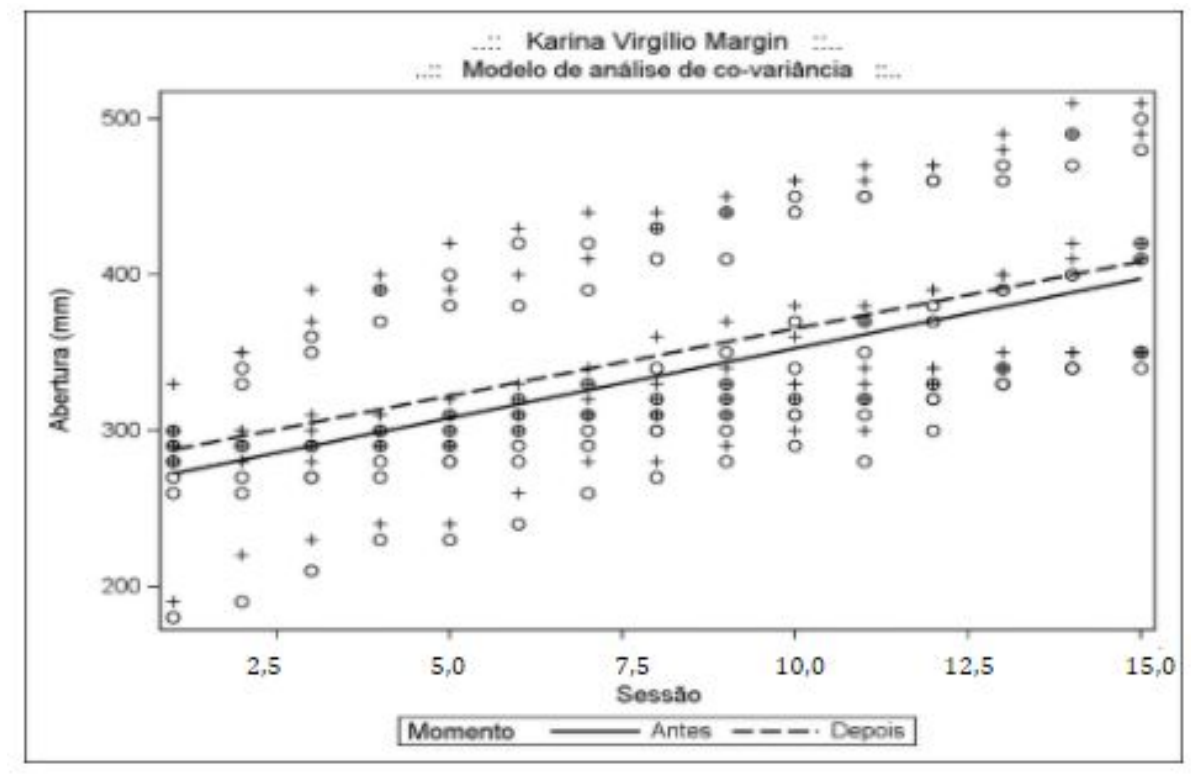

Figura 1. Modelo de análise de variância do efeito de Momento com a covariável sessão. Representação das 15 semanas de tratamento, sendo que todos os sujeitos estão representados em cada sessão com a abertura inicial (O) e abertura final (+). O sinal positivo contido no círculo significa que os sujeitos estão sobrepostos.

Na Figura 2 os dados revelaram diferença significativa após a intervenção mioterápica no grupo muscular dos supra-hioideos.

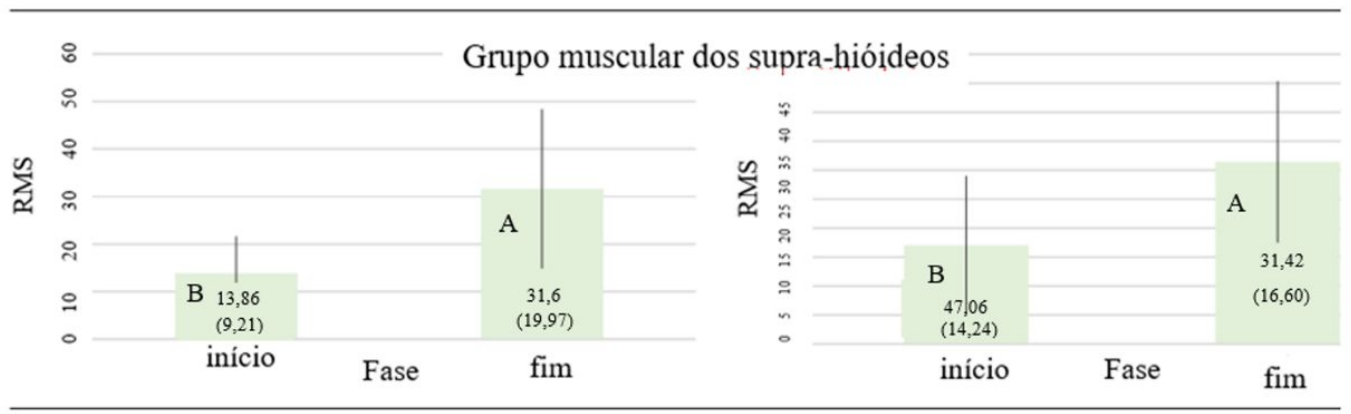

Figura 2. Média, desvio padrão, limites do intervalo de confiança da média (95\%) e teste $F$ para comparação das médias de RMS das fases, dos músculos supra-hioideos direito e esquerdo. Barras com letras iguais indicam médias que não diferem entre si no nível de significância de $5 \%$. 


\section{DISCUSSÃO}

A caracterização sociodemográfica dos nossos pacientes concordou com a previamente descrita na literatura: idade média de $\pm 57,5$ anos, predominância do gênero masculino $0^{9,23}$, com hábitos de tabagismo e/ou etilismo (Tabelas 1 e 2).

As manobras de crioalongamento associadas à massoterapia atuaram de forma significativa na liberação da abertura bucal nos pacientes após a neoplasia. 0 estudo foi conduzido em apenas oito pacientes, pois houve perdas no andamento do estudo. Cohen et al. ${ }^{23}$ refere que a redução de pacientes ao longo do tratamento, bem como a escassez de publicações sobre o tema, provavelmente têm uma relação com a área atingida pelo tumor, a cavidade oral e o pescoço 23 (Quadro 1). Essa região exige vasto cuidado em função da abundância de tecido mole e cavidades com espaços vazios, as quais, devido à suscetibilidade, quando estimuladas seja pela intervenção mioterápica ou pela própria liberação do trismo, favorecem a rápida proliferação da doença por serem consideradas ações dinâmicas. Com isso, medidas criteriosas foram adotadas antes de iniciar o tratamento para descartar possíveis focos do tumor, em que, diante da menor suspeita de recidiva, como presença de feridas ou vermelhidão, os casos foram interrompidos. Já em relação à efetividade do tratamento, a adoção de um delineamento estratégico também se fez necessário.

A crioterapia e a massoterapia foram realizadas não só no músculo masseter, mas também no grupo muscular dos supra-hioideos, responsáveis pela elevação e pelo abaixamento mandibular, respectivamente. Observou-se, em vários pacientes, que o tumor atingiu a região de assoalho da boca e mucosa jugal (Quadro 1), o que ocasionou intenso enrijecimento na área do pescoço. Como em $75 \%$ dos casos, o término da doença havia ocorrido entre um e 12 meses, e os pacientes ainda se queixavam de desconforto e até mesmo de dor, o tratamento foi então iniciado com a crioterapia (Quadro 1) (Tabela 2). As aplicações com gelo foram gradativas e intermitentes, por cerca de dois minutos ${ }^{4}$, fato que favoreceu o início do relaxamento muscular até que o resfriamento atingisse tecidos mais profundos e estimulasse os neurorreceptores sensoriais responsáveis pela analgesia ${ }^{16,23}$. Com o paciente mais confortável, foi então possível iniciar a massoterapia.

A massagem favoreceu não só a liberação da aderência decorrente do enrijecimento do tecido recentemente danificado pela doença como também do fibrosamento muscular devido a radioterapia e atrofia muscular. Felizmente, somente em dois casos (Quadro 1), o término da doença havia sido superior a dois anos, pois, distintamente do restante do corpo, cuja atrofia muscular é significativa após quatro meses de desuso, na região da face, esta ocorre em torno de 18 a 24 meses $^{1,4}$. Outro fator a ser considerado foi o tempo de tratamento. Na literatura, diversos estudos $^{24}$ relatam que a regeneração da fibra muscular diante de injúrias traumáticas depende de diversos fatores e pode demorar cerca de três meses. Ou seja, o restabelecimento não é imediato e exige que as manobras, ao longo do tratamento, também sejam gradativas tanto para o restabelecimento da fibra quanto para sua hipertrofia.

0 alongamento muscular também foi realizado seguindo uma série de critérios para que o reflexo de travamento, bem como o número reduzido de fibras, permitissem a abertura bucal 25 . A intensidade do movimento de alavanca realizado pelos dedos polegar e indicador entre a região alveolar dos dentes incisivos foi norteada pela percepção do paciente. A pressão foi mínima, mas deveria repercutir na articulação temporomandibular. Conforme a abertura bucal melhorava, os movimentos de alavanca avançavam para as regiões posteriores alveolares da cavidade bucal. Como, em média, a abertura bucal na sessão não excedia $2 \mathrm{~mm}$, tal parâmetro foi adotado, mesmo quando o paciente buscava abrir mais a boca. A sensibilidade tátil e a percepção do paciente foram decisivas para favorecer a abertura bucal sem ocasionar recidivas ou reinstalar o reflexo de travamento. 
0 aumento gradativo, mesmo que mínimo e sem recidivas ao longo do tratamento, comprova que a abertura bucal ocorreu (Figura 1), fato reforçado pela diferença significativa do grupo muscular dos supra-hioideos após a mioterapia (Figura 2). Os próprios pacientes, ao longo do tratamento, referiam que sentiam a boca mais liberada e que estavam conseguindo comer e realizar a higiene bucal com maior facilidade. $O$ fato de conseguirem voltar a mastigar com maior efetividade provavelmente também deve ter contribuído para a hipertrofia muscular.

No caso de doenças devastadoras como a neoplasia bucal, voltar a abrir a boca, mesmo não alcançando o padrão de normalidade, é muito significativo para o paciente. Poder exercer com maior conforto funções como mastigar e principalmente se expressar verbalmente provavelmente é um grande estímulo para o reinício de uma nova fase da vida.

Contudo, os resultados deste estudo devem ser confirmados em casuísticas maiores.

\section{REFERÊNCIAS}

1. Dijkstra PU, Sterken MW, Pater R, Spijkervet FKL, Roodenburg JLN. Exercise therapy for trismus in head and neck cancer. Oral Oncol. 2007 Apr;43(4):389-94. http://dx.doi.org/10.1016/j.oraloncology.2006.04.003. PMid:16979926.

2. Melo AUC, Rosa MRD, Agripino GG, Ribeiro CF. Informação e comportamento de cirurgiões-dentistas do Programa Saúde da Família de Aracaju a respeito de câncer bucal. Rev Bras Cir Cabeça Pescoço. 2008 Abr-Jun;37(2):114-9.

3. Santana JJRA, Zanin CR, Maniglia JV. Pacientes com câncer: enfrentamento, rede social e apoio social. Paidéia (Ribeirão Preto). 2008;18(40):371-84. http://dx.doi.org/10.1590/S0103$863 \times 2008000200013$.

4. Louise Kent M, Brennan MT, Noll JL, Fox PC, Burri SH, Hunter JC, et al. Radiation-induced trismus in head and neck cancer patients. Support Care Cancer. 2008 Mar;16(3):305-9. http://dx.doi.org/10.1007/s00520-007-0345-5. PMid:17965892.

5. Bensadoun RJ, Riesenbeck D, Lockhart PB, Elting LS, Spijkervet FKL, Brennan MT, Trismus Section, Oral Care Study Group, Multinational Association for Supportive Care in Cancer (MASCC)/International Society of Oral Oncology (ISO0). A systematic review of trismus induced by cancer therapies in head and neck cancer patients. Support Care Cancer. 2010 Aug;18(8):1033-8. http://dx.doi.org/10.1007/s00520-010-0847-4. PMid:20213237.

6. Dias M, de Souza SG, Della Justina LB. Tratamiento fisioterapeutico em paciente com trismo pósradioterapia. Revista Inspirar. Movimento \& Saúde. 2015 Abr-Jun;7(2):6-10.

7. Vissink A, Burlage FR, Spijkervet FKL, Jansma J, Coppes RP. Prevention and treatment of the consequences of head and neck radiotherapy. Crit Rev Oral Biol Med. 2003;14(3):213-25. http://dx.doi.org/10.1177/154411130301400306. PMid:12799324.

8. Barreira VG, Camargo EC. Efeitos fisiológicos e a aplicabilidade da crioterapia: uma atualização bibliográfica. Fisio Ter. 2003;7(36):22-8.

9. Kalliainen LK, Jejurikar SS, Liang LW, Urbanchek MG, Kuzon WM Jr. A specific force deficit exists in skeletal muscle after partial denervation. Muscle Nerve. 2002 Jan;25(1):31-8. http://dx.doi.org/10.1002/mus.1216. PMid:11754182.

10. Loorents V, Rosell J, Karlsson C, Lidbäck M, Hultman K, Börjeson S. Prophylactic training for the prevention of radiotherapy-induced trismus-a randomised study. Acta Oncol. 2014 Apr;53(4):530-8. http://dx.doi.org/10.3109/0284186X.2014.892211. PMid:24635110.

11. Bhatia KS, King AD, Paunipagar BK, Abrigo J, Vlantis AC, Leung SF, et al. MRI findings in patients with severe trismus following radiotherapy for nasopharyngeal carcinoma. Eur Radiol. 2009 Nov;19(11):2586-93. http://dx.doi.org/10.1007/s00330-009-1445-z. PMid:19504110.

12. Stubblefield MD, Manfield L, Riedel ER. A preliminary report on the efficacy of a dynamic jaw opening device (dynasplint trismus system) as part of the multimodal treatment of trismus in patients with 
head and neck cancer. Arch Phys Med Rehabil. 2010 Aug;91(8):1278-82.

http://dx.doi.org/10.1016/j.apmr.2010.05.010. PMid:20684911.

13. Bleakley C, McDonough S, MacAuley D. The use of ice in the treatment of acute soft-tissue injury: a systematic review of randomized controlled trials. Am J Sports Med. 2004 Jan-Feb;32(1):251-61. http://dx.doi.org/10.1177/0363546503260757. PMid:14754753.

14. Sampaio LR, Moura CVM, Resende MA. Recursos fisioterapêuticos no controle da dor oncológica: revisão de literatura. Rev Bras Cancerol. 2005;51(4):339-46.

15. Lewis T. Observations upon the reactions of the vessels of the human skin to cold. Heart. 1930;15:177208.

16. Cataldi JK, Pritchard KA, Hart JM, Saliba SA. Cryotherapy effects, part 2: time to numbness onset and numbness duration. Int J Athl Ther Train. 2013;18(5):26-8. http://dx.doi.org/10.1123/ijatt.18.5.26.

17. Knight KL. Cryostretch for muscle spasm. Phys Sportsmed. 1980 Apr;8(4):129. http://dx.doi.org/10.1080/00913847.1980.11710923. PMid:27432715.

18. Guirro R, Abib C, Máximo C. Os efeitos fisiológicos da crioterapia: uma revisão. Fisioter Pesqui. 1999;6(2):164-70. http://dx.doi.org/10.1590/fpusp.v6i2.79629.

19. Swenson C, Swärd L, Karlsson J. Cryotherapy in sports medicine. Scand J Med Sci Sports. 1996 Aug;6(4):193-200. http://dx.doi.org/10.1111/j.1600-0838.1996.tb00090.x. PMid:8896090.

20. Silva JC, de Souza Vieira C, de Souza LPL, de Araújo RC. Efeito da massoterapia nos sintomas da dor muscular tardia. Revista de Educação Física. 2010;79(148). http://dx.doi.org/10.37310/ref.v79i148.469.

21. Dijkstra PU, Kalk WWI, Roodenburg JLN. Trismus in head and neck oncology: a systematic review. Oral Oncol. 2004 Oct;40(9):879-89. http://dx.doi.org/10.1016/j.oraloncology.2004.04.003. PMid:15380165.

22. Hanson ML. Oral myofunctional therapy. Am J Orthod. 1978 Jan;73(1):59-67. http://dx.doi.org/10.1016/0002-9416(78)90102-1. PMid:271473.

23. Cohen EG, Deschler DG, Walsh K, Hayden RE. Early use of a mechanical stretching device to improve mandibular mobility after composite resection: a pilot study. Arch Phys Med Rehabil. 2005 Jul;86(7):1416-9. http://dx.doi.org/10.1016/j.apmr.2004.10.035. PMid:16003674.

24. Martins RS, Siqueira MG, da Silva CF, Pereira Plese JP. Mecanismos básicos da regeneração de nervos: artigo de revisão. Arquivos Brasileiros de Neurocirurgia: Brazilian Neurosurgery. 2005;24(1):20-5. http://dx.doi.org/10.1055/s-0038-1625458.

25. Laureano Filho JR, de Oliveira e Silva ED, Batista CI, Gouveia FM. The influence of cryotherapy on reduction of swelling, pain and trismus after third-molar extraction. J Am Dent Assoc. 2005 Jun;136(6):774-8, quiz 807. http://dx.doi.org/10.14219/jada.archive.2005.0261. PMid:16022042.

\section{CONFLITOS DE INTERESSE}

Os autores declaram não haver conflitos de interesse.

\section{*AUTOR PARA CORRESPONDÊNCIA}

Mirian Hideko Nagae, UNICAMP - Universidade Estadual de Campinas, Faculdade de Ciências Médicas, Rua Tessália Vieira de Camargo, 126, Barão Geraldo, 13084-971 Campinas, SP, Brasil, email: mnagae@fcm.unicamp.br

Recebido: Abril 22, 2020

Aprovado: Novembro 18, 2020 